\title{
Discriminant Analysis of Changes in Human Muscle Function When Interacting with an Assistive Aid
}

\author{
AUGUSTUS MORRIS, JR., MEMBER, IEEE, AND DANIEL W. REPPERGER, MEMBER, IEEE
}

\begin{abstract}
This paper examines the changes of human muscle function when the arm's simple antagonist tricep-bicep muscle pair is aided by a mechanical assistive device. The assistive aid changes how the muscles are used and this shift in muscle activity is determined objectively by using discriminant analysis. Surface EMG signals from the biceps and triceps provided the electrical signals. Four types of primitive arm movements were used in the detection of the muscle changes.
\end{abstract}

\section{INTRODUCTION}

$\mathrm{W}$ HEN humans interact with assistive controllers, changes in muscle function occur. This is a consequence of the use of assistive aids and here it is of interest to objectively quantify these changes by some available metric. This has been accomplished in other cases; for example, in [1]-[3] the control of a prosthetic arm was implemented with the help of pattern recognition techniques and classification procedures. Measuring EMG signals becomes one of the most convenient procedures to obtain information on physiological variables and has found wide application in studies on limb function [4] and neurogenic diseases [5]. Statistical techniques allow classification of data into groups that help identify particular arm motions [6], [7]. The discriminant tests [8] provide criteria for classification of empirical data as well as estimates of falsely classifying a result. In this study, the most basic type of primitive arm movements are investigated and consist of pure agonist and antagonist responses as well as some complex arm motions which relate to real world tracking motions.

Recently, interest has developed in the rehabilitation area [12]-[14] in restoring muscle function or control to paralyzed patients and also for prosthesis control. The use of such redirected actions using alternative muscle groups is referred to as using "synergistic signals" and has shown promise in the aid of those with muscular dysfunction.

\section{The Assistive Device}

The assistive device used in this study (termed "smart stick" in Fig. 1) was developed at Wright-Patterson Air Force Base and consists of a lateral displacement stick controller which can exert forces on the human's hand under computer control. A rack and pinion mechanical

Manuscript received June 24, 1986; revised November 2, 1987. The authors are with the Air Force Aerospace Medical Research Lab, Wright-Patterson Air Force Base, OH 45433. IEEE Log Number 8819589. assembly drives the stick and exerts forces on the hand. A piston in a sealed cylinder containing nitrogen gas pressure in each chamber is regulated by a current-pressure transducer which drives the rack and pinion system. A computer generates the electrical signal to control the current-pressure transducer. Thus, any measured signal (e.g., stick position output) can be processed through a transfer function and thus drive the stick with a dynamically changing force profile.

\section{The Performance Task}

The performance task chosen for this study was representative of a pilot flying an aircraft as illustrated in Fig. 2. The human observes a display between the target (denoted by the cross) and the plant output (denoted by the circle). The stick's output is a voltage signal proportional to the stick's displacement and this voltage signal becomes the input to the system being controlled (plant = $k / s$ or a simple integrator). When the stick is passive, the loop involving $K s$ is absent, i.e., there is no force signal interacting with the human operator's hand. In the active stick case, however, the plant output is multiplied by $K s$ and fed to the force summer; thus, a force signal proportional to plant velocity is obtained.

\section{THE EXPERIMENT}

Seven healthy male subjects between the ages of 20-39 years (active duty Air Force military personnel) participated in this experiment. They were involved in three days of training on the tracking performance task and, on the fourth day, data were collected. Each subject had four silver/silver chloride surface electrodes (a differential pair) placed on the biceps and triceps and a reference electrode located on the forearm. These electrodes were all placed on the subject's right arm. The subject's skin resistance was required to be less the $20000 \Omega$ before the signal could be recorded. The EMG signals were then full-wave rectified, high-pass filtered to remove limb artifact motion (less than $2 \mathrm{~Hz}$, see Neilson [9]), and integrated with respect to a reset time of $T=160 \mathrm{~ms}$ (Fig. 3). The use of this IEMG signal has been found to be the best indicator of specific muscle activity in prosthesis work [1], [2], [10] when compared to zero crossings, peak values, rectified average value, and other indicators. The electrodes were placed $2 \mathrm{~cm}$ apart on each subject to ensure the same bandwidth of the bipolar signal (cf. Zipp [11]). 


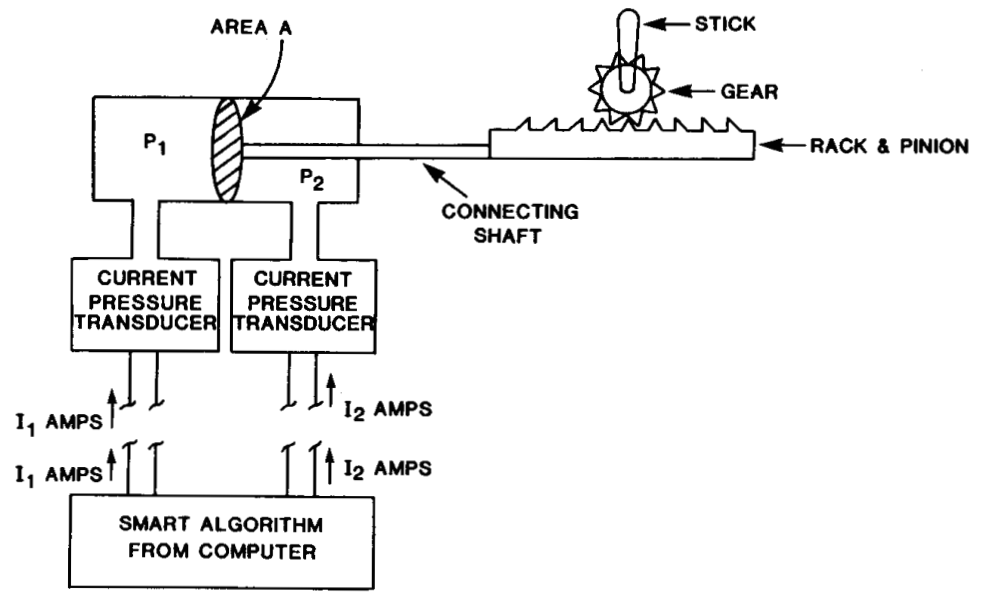

Fig. 1. The electromechanical device: The "smart stick."

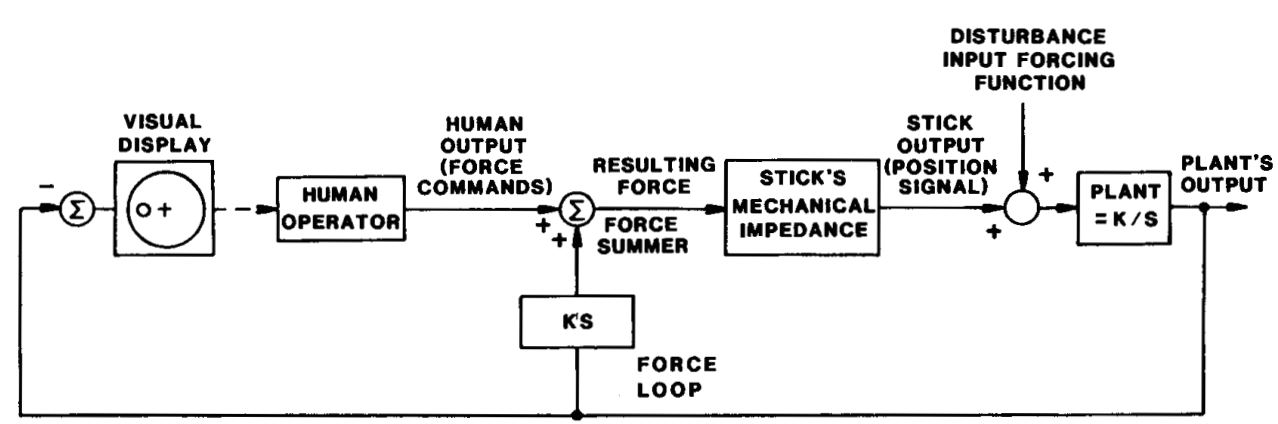

Fig. 2. The man-machine system.

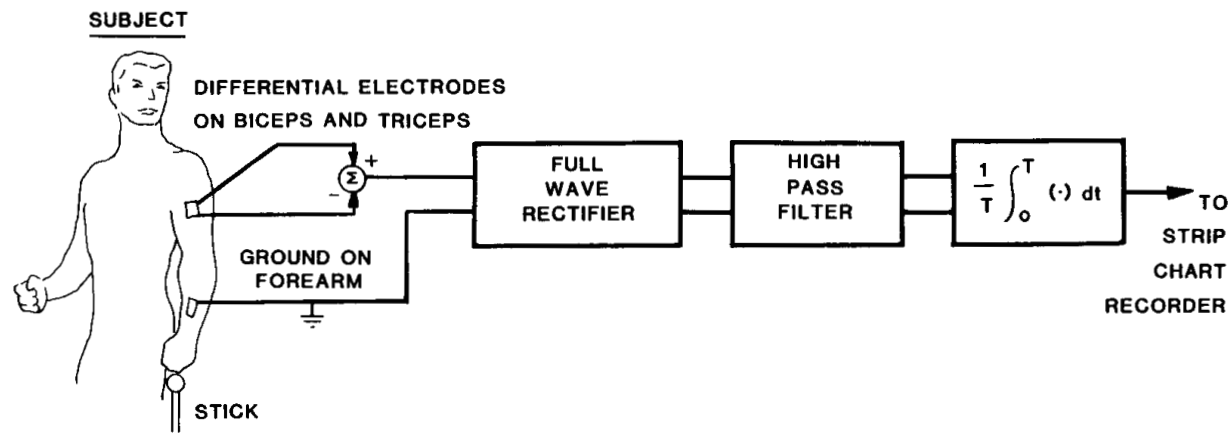

Fig. 3. The EMG signal processing procedure.

Each day the subjects were required to perform ten trials each of bicep curls (pure flexor movement), tricep curls (pure extensor movement) [Fig. 4(a) and (b)], as well as tricep tracking movements (lateral arm movements) [Fig. 5(a) and (b)], and bicep tracking movements (lateral arm movements). The bicep and tricep curl primitive movements used a small weight of approximately $500 \mathrm{~g}$. The choice of the tricep and bicep tracking movements provided a link between the pure flexor and extensor movements (bicep and tricep curls) and real world tracking behavior. For the tracking movements considered here, the elbow was fixed and supported for lateral arm excursions in an effort to preclude small arm rotations which may add some artifact in this experiment.

\section{The ObJective of THE EXPERIMENT}

It is desired to characterize muscle responses into features or similar characteristics. We first define the four arm motions in Figs. 4(a) and (b) and 5(a) and (b) as primitives. In particular, the pure bicep and tricep responses are the primitive agonist and antagonist responses least effected by artifact motion. Fig. 6(a) illustrates the empirical data from the pure bicep and tricep arm curls selected from one subject to form the primitives for the 


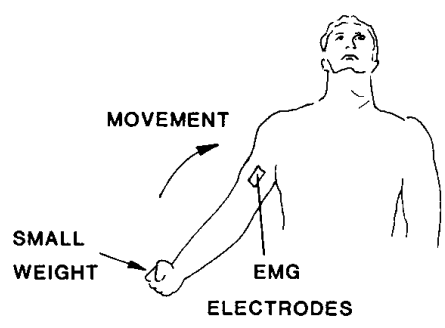

(a)

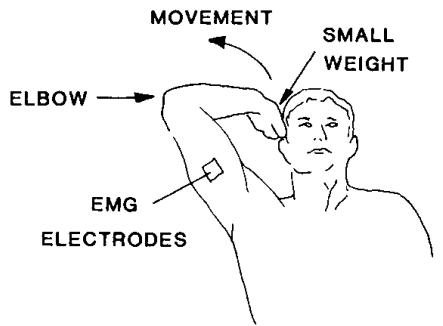

(b)

Fig. 4. (a) Bicep curls (pure flexor movement). (b) Tricep curls (pure extensor movement).

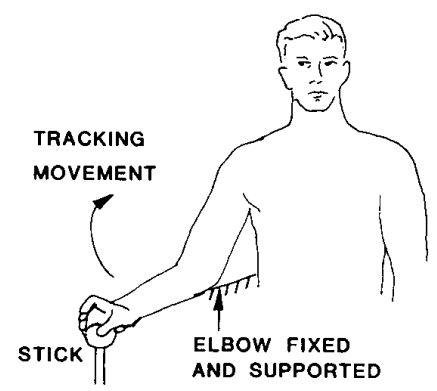

(a)

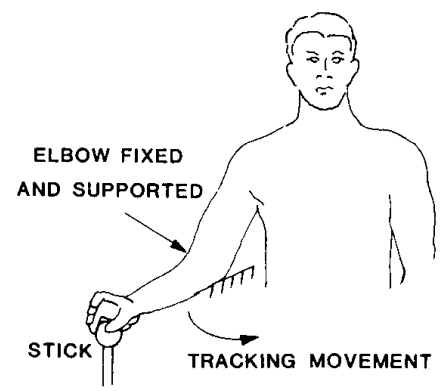

(b)

Fig. 5. (a) Tricep tracking movement (lateral arm movement). (b) Bicep tracking movement (lateral arm movement).

first feature selection space. In Fig. 6(b), the second feature space includes the arm movements for tracking biceps and triceps as the primitives. Fig. 7(a) and (b) illustrates the scatter plot of the actual empirical pure bicep and tricep curl data (primitives). Using a feature space approach as indicated in Fig. 7(a) and (b) with IEMG signals as the respective axes, it is necessary that there exist large distance in this space to separate these empirical data involving the different primitive movements.

\section{Data Analaysis From One Subject}

Since analysis of data involving muscle response is extremely subject dependent, analysis of the experimental conditions (or groups) within a subject, across the experimental conditions is first conducted. This is a common procedure for studying data in the prosthesis area. After data are analyzed within a subject, data are then analyzed across subjects to see what can be said in general for a population of subjects. Using the integrated absolute value of the surface EMG (IEMG) signal, data were tabulated on the pure bicep and tricep axes using the IEMG data. Table I illustrates the results from one subject. From Table I, differences in median and mean values of IEMG for the tracking bicep versus bicep curl on the bicep axes are observed. We also see differences in median and mean values between tracking tricep and tricep curl on the tricep axis.

There are several methods of analysis using these data from one subject. To test between groups made up of the populations of the four primitive movements, a ranking order test was used. From the Kruskal-Wallis one-way layout test (using Chi-square large sample approximation), a significant difference between the groups was found for the biceps [Chi-square $(5)=243, P=0.0001$ ] and triceps [Chi-square (5) $=361, P=0.0001$ ]. To determine pairwise differences between the groups, the Wilcoxon signed rank test was performed (using a $T$ test large sample approximation) with results ( $P$ values ) (Table II).

From Table II, the tracking bicep primitive was compared to the bicep curl primitive and these populations differ $(P=0.0002$ on the bicep axes and $P=0.0001$ on the tricep axes) as shown in Table I. Thus, the primitives sharply differed from one another in both cases $[P$ less than 0.0002 ] for all entries.

\section{Data Analysis Across the Seven Subjects}

Data are now analyzed for all seven subjects in the experiment. In the earlier phase of the analysis, the actual data used in the analysis of variance was determined by differences between the four groups. The term "group" referred to the four primitive arm motions. In this phase of the analysis, a discriminant determination was used to ascertain the closeness of the primitives to each other.

In a two-way analysis of variance with group as a fixed factor and subject as a random factor, if the same number of IEMG measurements were available in each cell, only the means of the cells would be used to test for differences in group. That is, only one number would represent each subject and group. The data obtained here, however, ranged from a distribution appearing normal to a distribution that was strongly skewed towards 0 . Therefore, the median was assumed to be a better measure of central 


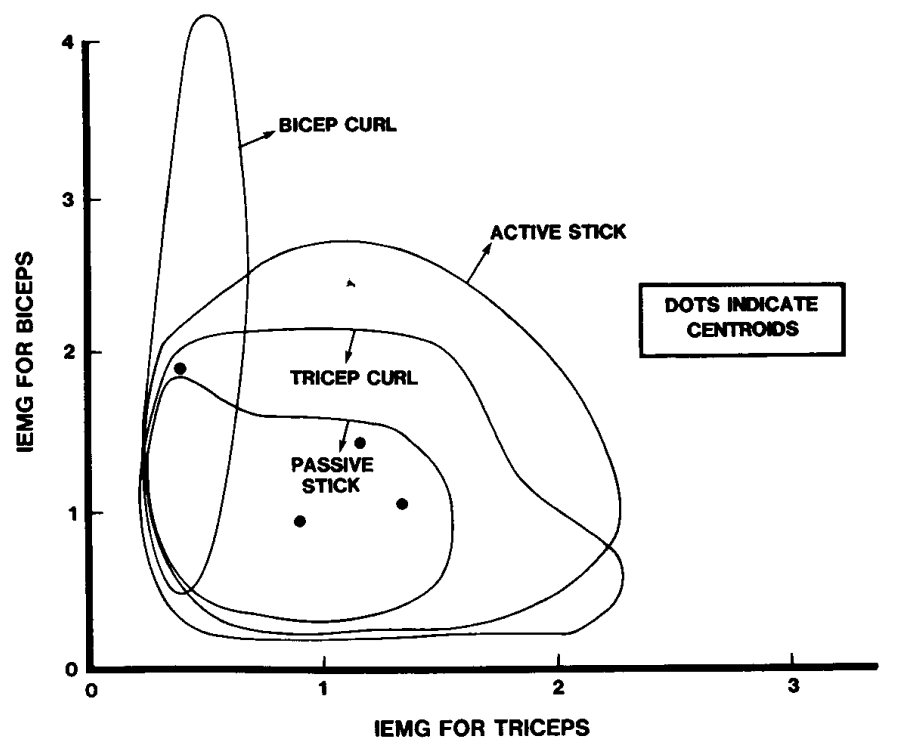

(a)

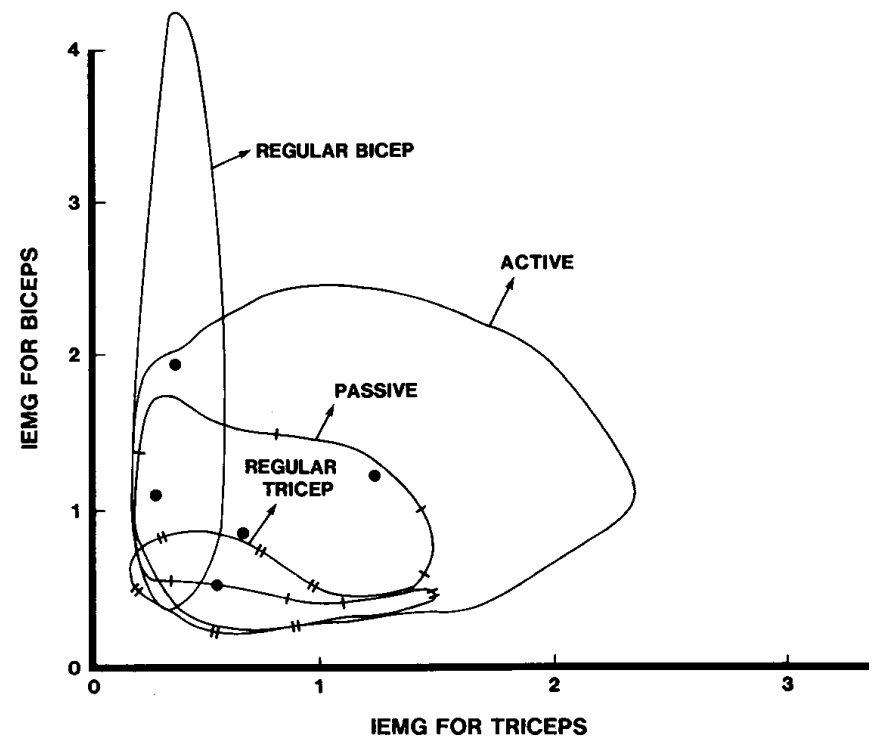

(b)

Fig. 6. (a) Curls as primitives. (b) Tracking movements as primitives.

tendency than the mean and it was used to represent each subject and group in an analysis of variance.

An analysis of variance performed on the medians found that equal error variance for the four groups could not be assumed. To adjust for this problem, a natural log transformation of the medians was used for the analysis. Table III gives the $P$ values for pairwise comparisons among the groups using $T$ tests.

It is of interest to compare the four primitives to each other using data averaged across seven subjects. To illustrate these results more explicitly, Table IV gives the $P$ values involving interaction with the actual median values.
The terms Passive (no force loop) and Active (with the force loop) refer to modes of operation of the assistive device used. In this table, if mean values of the medians of these variables are underlined, this indicates that they were not significantly different using a 0.01 per comparison error level. The means and standard deviations of subjects represent the original data. Thus, from Table V it is seen that the magnitude of the median triceps and biceps do not differ significantly (at a $P=0.01$ level) from each other but show differences between most of the primitives.

The question arises whether the percent of observations for the active and passive stick data are closer in a dis- 


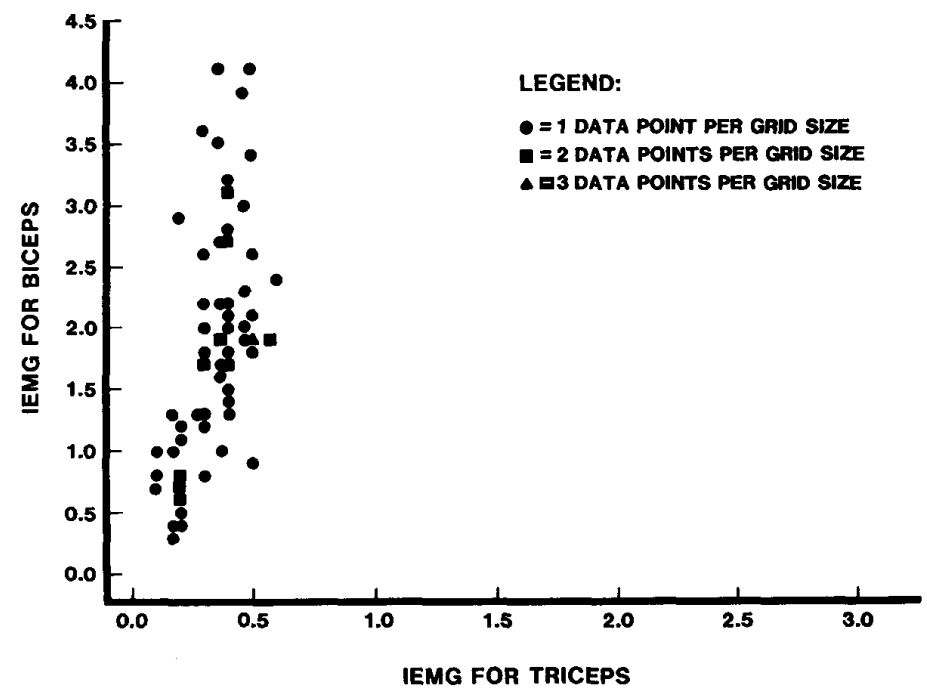

(a)

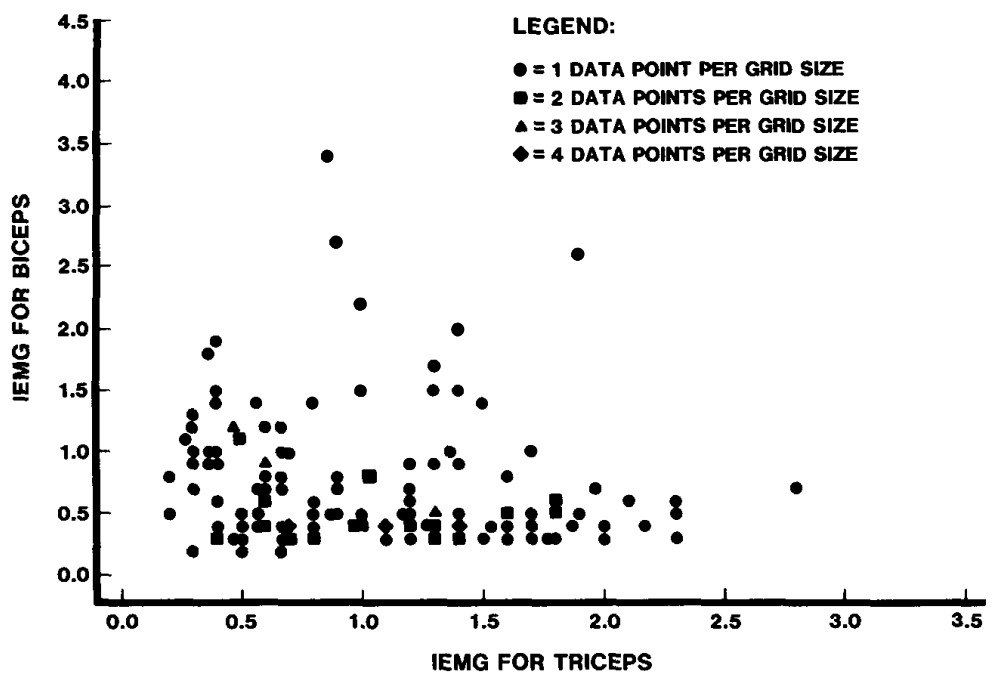

(b)

Fig. 7. (a) Bicep curls (empirical data). (b) Tricep curls (empirical data).

TABLE I

Mean, Median, and STD For IEMG's for the Four Primitives and One Subject

\begin{tabular}{llllllll}
\hline \hline & \multicolumn{3}{c}{ Biceps } & & \multicolumn{3}{c}{ Triceps } \\
\cline { 2 - 3 } \cline { 7 - 8 } $\begin{array}{l}\text { Experimental } \\
\text { Condition }\end{array}$ & Mean & Median & Std. & Mean & Median & Std. \\
\hline Tracking bicep & 1.37 & 1.05 & 1.00 & & 0.26 & 0.25 & 0.09 \\
Tracking tricep & 0.31 & 0.27 & 0.17 & & 0.56 & 0.50 & 0.27 \\
Bicep curl & 1.85 & 1.82 & 0.94 & 0.34 & 0.35 & 0.12 \\
Tricep curl & 0.71 & 0.50 & 0.52 & & 1.03 & 0.95 & 0.55
\end{tabular}

tance sense to one primitive or to another. This would indicate a shift of actual muscle activity from one primitive motion to another primitive motion. This is truly a relative measure. The answer to this question appears in Table V. The generalized squared distance to the primi-
TABLE II

Comparison Between Groups-One Subject

\begin{tabular}{lccc}
\hline Primitives & $\begin{array}{c}\text { Tracking } \\
\text { Tricep }\end{array}$ & $\begin{array}{c}\text { Bicep } \\
\text { Curl }\end{array}$ & $\begin{array}{c}\text { Tricep } \\
\text { Curl }\end{array}$ \\
\hline T. bicep & 0.0001 & 0.0002 & 0.0001 \\
& 0.0001 & 0.0001 & 0.0001 \\
T. tricep & & 0.0001 & 0.0001 \\
& & 0.0001 & 0.0001 \\
Bicep curl & & & 0.0001 \\
& & & 0.0001 \\
\hline Legend: Bicep Axis & & \\
& Tricep Axis & &
\end{tabular}

tives for both the active and passive stick condition was determined using percents (Table V).

Table $\mathrm{V}$ indicates that when comparing the passive stick 
TABLE III

$P$ Values For Pairwise Comparisons-Across Seven Subjects

\begin{tabular}{lccc}
\hline \hline Primitives & $\begin{array}{c}\text { Tracking } \\
\text { Tricep }\end{array}$ & $\begin{array}{c}\text { Bicep } \\
\text { Curl }\end{array}$ & $\begin{array}{c}\text { Tricep } \\
\text { Curl }\end{array}$ \\
\hline Tracking bicep & 0.0001 & 0.0014 & 0.0112 \\
& 0.0001 & 0.1417 & 0.0001 \\
Tracking tricep & & 0.0001 & 0.0003 \\
& & 0.0001 & 0.0607 \\
Bicep curl & & & 0.0001 \\
& & & 0.0001 \\
\hline
\end{tabular}

Legend: $P$ Values for Median Biceps

$P$ Values for Median Triceps

TABLE IV

Median Bicep IEMG's-Averaged Across Seven Subjects Median BICEPS

\begin{tabular}{|c|c|c|c|c|c|c|}
\hline & $\begin{array}{c}\text { Tracking } \\
\text { Tricep }\end{array}$ & $\begin{array}{c}\text { Tricep } \\
\text { Curl }\end{array}$ & Passive & $\begin{array}{c}\text { Tracking } \\
\text { Bicep }\end{array}$ & Active & $\begin{array}{c}\text { Bicep } \\
\text { Curl }\end{array}$ \\
\hline \multirow[t]{3}{*}{$\begin{array}{l}\text { Mean } \\
\pm \text { STD }\end{array}$} & $\begin{array}{l}0.21 \\
0.04\end{array}$ & $\begin{array}{l}0.61 \\
0.45\end{array}$ & $\begin{array}{l}0.92 \\
0.59\end{array}$ & $\begin{array}{l}0.97 \\
0.41\end{array}$ & $\begin{array}{l}1.19 \\
1.02\end{array}$ & $\begin{array}{l}2.24 \\
1.47\end{array}$ \\
\hline & \multicolumn{6}{|c|}{ Median Triceps } \\
\hline & $\begin{array}{c}\text { Tracking } \\
\text { Bicep }\end{array}$ & $\begin{array}{c}\text { Bicep } \\
\text { Curl }\end{array}$ & Passive & Active & $\begin{array}{l}\text { Tracking } \\
\text { Tricep }\end{array}$ & $\begin{array}{c}\text { Tricep } \\
\text { Curl }\end{array}$ \\
\hline $\begin{array}{l}\text { Mean } \\
\pm S T D\end{array}$ & $\begin{array}{l}0.17 \\
0.06\end{array}$ & $\begin{array}{l}0.26 \\
0.12\end{array}$ & $\begin{array}{l}0.46 \\
0.23\end{array}$ & $\begin{array}{l}0.79 \\
0.68\end{array}$ & $\begin{array}{l}0.79 \\
0.43\end{array}$ & $\begin{array}{l}1.35 \\
1.01\end{array}$ \\
\hline
\end{tabular}

TABLE V

Percent Closeness To Each Group-One Subject

\begin{tabular}{lrcccc}
\hline \hline Group & Total & $\begin{array}{c}\text { Tracking } \\
\text { Bicep }\end{array}$ & $\begin{array}{c}\text { Tracking } \\
\text { Tricep }\end{array}$ & $\begin{array}{c}\text { Bicep } \\
\text { Curl }\end{array}$ & $\begin{array}{c}\text { Tricep } \\
\text { Curl }\end{array}$ \\
\hline Active & 247 & $20 \%$ & $80 \%$ & $14 \%$ & $86 \%$ \\
Passive & 92 & $37 \%$ & $63 \%$ & $35 \%$ & $65 \%$ \\
\hline
\end{tabular}

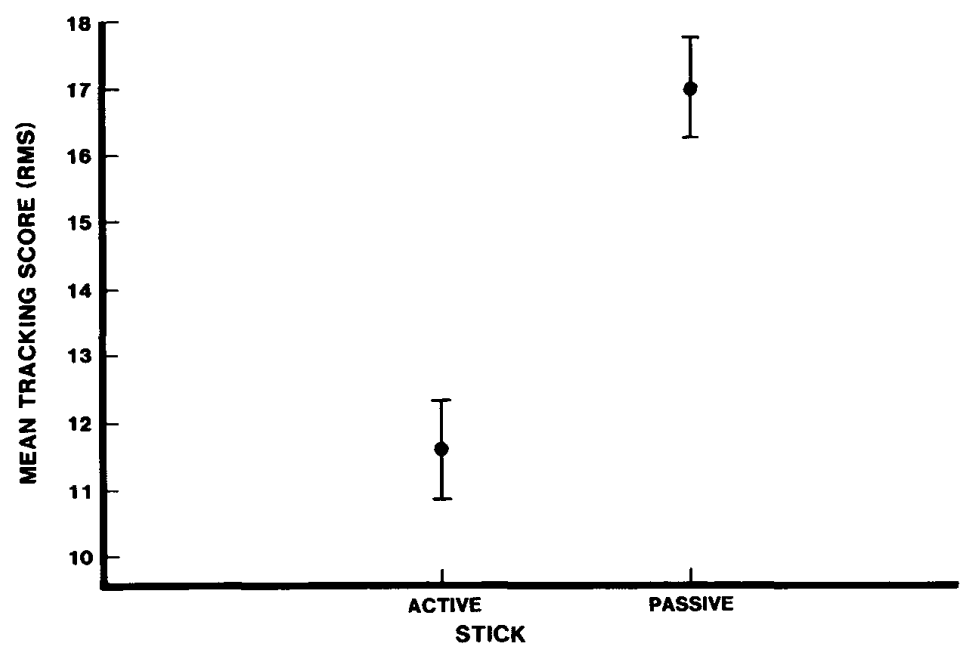

Fig. 8. Comparison of erms tracking scores for active stick versus passive stick ( 95 percent confidence intervals). 
TABLE VI

Performance Data Across Subjects (erms Values)

\begin{tabular}{lcc}
\hline \hline & \multicolumn{2}{c}{ Stick } \\
\cline { 2 - 3 } & Active & Passive \\
\hline Mean \pm STD of Subjects & $11.57 \pm 0.95$ & $17.02 \pm 1.07$ \\
\hline
\end{tabular}

condition versus the active stick experimental condition, a substantial shift occurs in the bicep motions relative to tricep motion; a shift of 17 percent when using tracking biceps and tracking triceps as primitives; a shift of 21 percent when considering the bicep and tricep curls as the primitives. Thus, the assistive device did change the muscle function and is essentially increased tricep activity approximately 20 percent and decreased the bicep activity by the same amount for this one subject. This assistive device does appear to alter the muscle function used.

Across subjects, this analysis showed that the active stick group used more tricep activity because both IEMG's increase, but a greater increase occurred with the triceps. More variability resulted across subjects.

\section{ViII. Tracking Performance Measurements}

One measure of the usefulness of an assistive device occurs if the total man-machine system performs better with the device as compared to without the device. To determine the effectiveness of the assistive aid, rms (root mean square) tracking error was calculated during the 85 $s$ run. Fig. 8 illustrates the ensemble data across all seven subjects on day four. The $F$ test found a significant difference in the tracking scores between the sticks $[F(1,6)$ $=154, P=0.0001]$ with means shown in Table VI.

Using root mean square tracking error, the lower the score, the better the performance. These data demonstrate that the assistive device did indeed improve the man-machine performance. These data were averaged across seven subjects, so that the performance results are not subject dependent, but apply across a general population of subjects. Since this performance change is correlated with the muscle changes in Table $\mathrm{V}$, it follows that an improvement in tracking performance is correlated to this change in muscle function.

\section{Summary and Conclusions}

Using data from an empirical study on primitive arm movements and IEMG signals, data are analyzed using a feature selection space to characterize empirical data. Discriminant analysis is used to classify data into features of response characteristics.

Bicep and tricep EMG's can be clearly distinguished from one another in the feature selection space both within a subject and across subjects. Using the assistive device studied in this paper gives rise to specific changes in muscle function which can be correlated with improved tracking performance.

\section{REFERENCES}

[1] S. Lee and G. N. Saridis, "The control of a prosthetic arm by EMG pattern recognition,"'IEEE Trans. Automat. Contr., vol. AC-29, pp. 290-302, Apr. 1984

[2] D. Graupe and W. K. Cline, "Functional separation of EMG signals via ARMA identification methods for prothesis control purposes," IEEE Trans. Syst, Man, Cybern., vol. SMC-5, Mar. 1975.

[3] G. N. Saridis and H. E. Stephanou, "A hierarchical approach to the control of a prosthetic arm," IEEE Trans. Syst., Man, Cybern., vol. SMC-7, pp. 407-420, June 1977.

[4] P. C. Doerschuk, D. E. Gustafson, and A. S. Willsky, "Upper extremity limb function discrimination using EMG signal analysis," IEEE Trans. Biomed. Eng., vol. BME-30, pp. 18-28, Jan. 1983.

[5] C. Berzuini, M. M. Figini, and L. Bernardinelli, "Evaluation of the effectiveness of EMG parameters in the study of neurogenic diseases-A statistical approach using clinical and simulated data," IEEE Trans. Biomed. Eng., vol. BME-32, pp. 15-27, Jan. 1985.

[6] D. C. Dening, F. G. Gray, and R. M. Haralick, "Prosthesis control using a nearest neighbor electromyographic pattern classifier," IEEE Trans. Biomed. Eng., vol. BME-30, pp. 356-360, June 1983.

[7] D. W. Gilstad and K. S. Fu, "Two-dimensional adaptive model of a human controller using pattern recognition," IEEE Trans. Syst., Man, Cybern., pp. 261-266, July 1971

[8] D. W. Repperger, S. L. Ward, E. J. Harzell, B. C. Glass, and W. Summers, "An algorithm to ascertain critical regions of human tracking ability," IEEE Trans. Syst., Man, Cybern., vol. SMC-9, pp. $183-$ 196. Apr. 1979.

[9] P. D. Neilson, "Interaction between voluntary contraction and tonic stretch reflex transmission in normal and spastic patients," J. Neurol. Neurosur. Psych., vol. 35, p. 853, 1972.

[10] G. L. Gottlieb and G. C. Agarwal, "Filtering of electromyographic signals," Amer. J. Phys. Med., vol. 33, pp. 365, 1970.

[11] P. Zipp. "Effect of electrode parameters on the bandwidth of the surface EMG power-density spectrum,"' Med. Biol. Eng. Comp., vol. 16, pp. $537,1978$.

[12] A. Karlj, T. Bajd, R. Turk, J. Kreajnik, and H. Benko, "Gait restoration in paraplegic patients: A feasibility demonstration using multichannel surface electrode/FES," J. Rehab. Res. Develop., vol. 18, no. 2, pp. 3-11, 1981

[13] R. M. Glaser, J. A. Gruner, S. D. Feinberg, and S. R. Collins, "Locomotion via paralyzed leg muscles: Feasibility study for a leg-propelled vehicle," J. Rehab. Res. Develop., vol. 20, no. 1, pp. 87-92, 1983.

[14] J. A. Doubler and D. S. Childress, "Design and evaluation of a prosthesis control system based on the concept of extended physiological proprioception," J. Rehab. Res. Develop., vol. 21 , no. 1, pp. 1931,1984 .

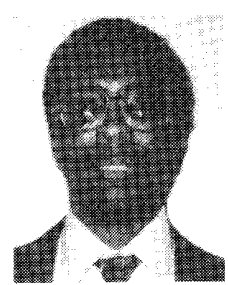

Augustus Morris, Jr. (S'81-M $81-S^{\circ} 81-M^{\prime} 85-$ $S^{\prime} 86-M^{\circ} 86$ ) was born on May 26, 1959 in Battle Creek, MI. He received the B.S. degree in biomedical engineering from Wright State University. Dayton, OH. in 1981 .

Presently, he is a doctoral candidate in the Biomedical Sciences program at wright State University. He is also an Instructor of Manufacturing Engineering at Central State University. Wilberforce, $\mathrm{OH}$. His current research interest is in the design and control of teleoperated robotic manipulators using adaptive control, fuzzy control, and artificial intelligence techniques. He was a National Science Foundation Fellow and presently has submitted two patent applications.

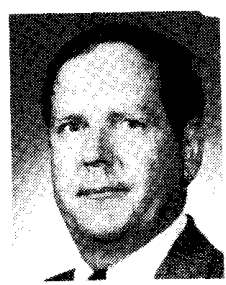

Daniel W. Repperger ( $S^{\prime} 66-S^{\prime} 69-M^{\prime} 74$ ) was born in 1942 in Charlestown, SC. He received the B.S.E.E. and M.S.E.E. degrees from Rensselaer Polytechnic Institute, Troy, NY, in 1967 and 1968 , respectively, and the Ph.D. degree in electrical engineering from Purdue University. Lafayette, IN. in 1973.

From 1973 to 1974 he held a National Research Council Postdoctoral Fellowship at the Acrospace Medical Research Laboratory, WrightPatterson Air Force Base. OH. Presently, he is a civil servant at the Armstrong Aerospace Medical Research Laboratory. His areas of interest include man-machine systems, biomedical applications. and robotics. 